\title{
Thermodynamic and dynamic contributions to future changes in regional precipitation variance: focus on the Southeastern United States
}

\author{
Laifang Li • Wenhong Li
}

Received: 26 February 2014/ Accepted: 13 June 2014

(C) Springer-Verlag Berlin Heidelberg 2014

\begin{abstract}
The frequency and severity of extreme events are tightly associated with the variance of precipitation. As climate warms, the acceleration in hydrological cycle is likely to enhance the variance of precipitation across the globe. However, due to the lack of an effective analysis method, the mechanisms responsible for the changes of precipitation variance are poorly understood, especially on regional scales. Our study fills this gap by formulating a variance partition algorithm, which explicitly quantifies the contributions of atmospheric thermodynamics (specific humidity) and dynamics (wind) to the changes in regionalscale precipitation variance. Taking Southeastern (SE) United States (US) summer precipitation as an example, the algorithm is applied to the simulations of current and future climate by phase 5 of Coupled Model Intercomparison Project (CMIP5) models. The analysis suggests that compared to observations, most CMIP5 models ( $\sim 60 \%)$ tend to underestimate the summer precipitation variance over the SE US during the 1950-1999, primarily due to the errors in the modeled dynamic processes (i.e. large-scale circulation). Among the 18 CMIP5 models analyzed in this study, six of them reasonably simulate SE US summer precipitation variance in the twentieth century and the underlying physical processes; these models are thus applied for mechanistic study of future changes in SE US summer precipitation variance. In the future, the six models collectively project an intensification of SE US summer precipitation variance, resulting from the combined effects of atmospheric thermodynamics and
\end{abstract}

L. Li $(\bowtie) \cdot$ W. Li

Earth and Ocean Sciences, Nicholas School of the Environment and Earth Sciences, Duke University, 322 Old Chem. Bldg, P.O. Box 90227, Durham, NC 27708, USA

e-mail: laifang.li@duke.edu dynamics. Between them, the latter plays a more important role. Specifically, thermodynamics results in more frequent and intensified wet summers, but does not contribute to the projected increase in the frequency and intensity of dry summers. In contrast, atmospheric dynamics explains the projected enhancement in both wet and dry summers, indicating its importance in understanding future climate change over the SE US. The results suggest that the intensified SE US summer precipitation variance is not a purely thermodynamic response to greenhouse gases forcing, and cannot be explained without the contribution of atmospheric dynamics. Our analysis provides important insights to understand the mechanisms of SE US summer precipitation variance change. The algorithm formulated in this study can be easily applied to other regions and seasons to systematically explore the mechanisms responsible for the changes in precipitation extremes in a warming climate.

Keywords Southeastern US summer precipitation variance - Atmospheric hydrological cycle - Variance partition algorithm . Thermodynamic and dynamic components $\cdot$ Extreme precipitation

\section{Introduction}

The occurrence and severity of precipitation extremes are tightly associated with the variance of precipitation ${ }^{1}$ (e.g., Katz and Brown 1992). As climate warms, more frequent and severe extremes have been observed and verified by

\footnotetext{
${ }^{1}$ The variance of precipitation measures how precipitation records are spread out. High variance in precipitation shows a higher probability of precipitation to deviate from its climatological mean, indicating an increasing likelihood of both dry and wet extremes in precipitation.
} 
models (e.g., Karl and Knight 1998; Kharin and Zwiers 2000; Trenberth et al. 2003; Trenberth 2011; Mahajan et al. 2012). The changes in climate extremes are attributable to the enhancement of precipitation variance resulting from the acceleration of hydrological cycle (e.g., Giorgi and Francisco 2000; Yang et al. 2003; Held and Soden 2006; Huntington 2006; Seager et al. 2007, 2010; Lau et al. 2013). In addition, the changes in the hydrological cycle can be either thermodynamic or dynamic (Seager et al. 2007,2010 ). On the one hand, the warming of the troposphere tends to increase atmospheric moisture content, thermodynamically intensify the atmospheric hydrological cycle (e.g., Allen and Ingram 2002; Dai 2006; Held and Soden 2006; Santer et al. 2007; O'Gorman and Schneiderb 2009; Seager et al. 2010, 2012; Sherwood 2010). On the other hand, projected changes in atmospheric circulation can also dynamically modulate the hydrological cycle (e.g., Vecchi et al. 2006; Cai et al. 2012; Li et al. 2012a; Scheff and Frierson 2012; Feng and Fu 2013).

Changes in the thermodynamic and dynamic components of atmospheric hydrological cycle have already shifted the climatological patterns of regional precipitation, which have been widely studied (e.g., Dairaku and Emori 2006; O'Gorman and Schneiderb 2009; Skific et al. 2009; Kawase et al. 2010). However, the relative contributions of atmospheric thermodynamics and dynamics to changes in precipitation variance are poorly understood, which hampers the mechanistic interpretation of regional climate change, in particular of extreme events.

More importantly, existing analysis methods cannot separate the thermodynamic and dynamic contributions to the variance of hydrological cycle without strict assumptions. For example, Seager et al. (2012) inferred thermodynamic and dynamic contributions to variance changes in the global hydrological cycle, by assuming that the El Niño Southern Oscillation (ENSO) dominates the variance of the hydrological cycle. However, this assumption would not be applicable in regions where the ENSO induced variance is insignificant, particularly in the summer (e.g., Clark et al. 2000; Kushnir et al. 2010; Li et al. 2013b). Thus, a more generalized method, explicitly quantifying the thermodynamic and dynamic contributions to precipitation variance change, is needed to identify the causes of hydroclimatic change especially on a regional scale.

This study proposes a generalized variance partition algorithm to advance the mechanistic study of global and regional hydrological cycles. This algorithm is applied to summer precipitation over the Southeastern United States (SE US), a fast developing region of the nation, where the variance of summer precipitation has significantly intensified in recent decades and the resultant extreme events have risked regional agriculture, ecology, and water management (e.g. Manuel 2008; Martinez et al. 2009; Gotvald and
McCallum 2010; Wang et al. 2010; Li et al. 2011). Furthermore, climate models suggest that SE US summer precipitation variance will intensify by the end of the twenty-first century, indicating a higher likelihood of future summer climate extremes ( $\mathrm{Li}$ et al. 2013a; Wuebbles et al. 2013). Thus, there is a pressing need to understand the processes and causes responsible for the projected changes in summer precipitation variance.

By implementing the variance partition algorithm to SE US summertime hydroclimate as simulated by the phase 5 of Coupled Model Intercomparison Project (CMIP5) models and reanalysis datasets, this study aims to achieve two scientific objectives: (1) to explore the processes and factors that constrain CMIP5 models' skill in simulating the SE US summer precipitation variance; and (2) to quantify thermodynamic and dynamic contributions to the enhanced SE US summer precipitation variance in a warming climate, upon which mechanisms responsible for regional precipitation change are provided.

The rest of the manuscript is organized as follows. In Sect. 2, the data used to calculate the regional atmospheric hydrological processes are presented. The variance partition algorithm is formulated in Sect. 3. The algorithm is then implemented in Sect. 4 to diagnose the thermodynamic and dynamic contributions to the SE US summer precipitation variance simulated by CMIP5 models in current climate. In Sect. 5, the algorithm is applied to assess the mechanisms of future intensification of SE US summer precipitation variance. Conclusions are given in Sect. 6.

\section{Observation, reanalysis datasets, and CMIP5 model output}

In this study, the observed precipitation, multiple reanalysis datasets, and the output from CMIP5 models are applied to quantify the atmospheric branch of the hydrological cycle over the SE US and to project future changes in regional hydroclimate. The observed precipitation is derived from the ensemble of three datasets including the National Oceanic and Atmospheric Administration (NOAA)'s PRECipitation REConstruction over Land (Prec/L) datasets (Chen et al. 2002); NOAA Climate Prediction Center (CPC) US unified precipitation (Higgins et al. 2000) and the Global Precipitation Climatology Centre (GPCC) monthly precipitation dataset (Rudolf et al. 2005), following Li et al. (2013a).

The variables used to quantify the atmospheric hydrological cycle are specific humidity, wind velocity, and surface pressure. These variables are adopted from multiple reanalysis datasets to ensure the robustness of the analysis results: National Centers for Environmental Prediction 
(NCEP)/National Center for Climate Research (NCAR) (Kalnay et al. 1996), European Center for Medium-Range Weather Forecasting (ECMWF) 40 year Reanalysis (ERA40) (Uppala et al. 2005), Japanese 25-year Reanalysis Project (JRA-25) (Onogi et al. 2007) and NCEP North American Regional Reanalysis (NARR) (Mesinger et al. 2006). Previous studies suggest that the four datasets are consistent in characterizing SE US summertime atmospheric hydrological processes, as well as quantifying their contributions to SE US summer precipitation variance ( $\mathrm{Li}$ et al. 2013b). In this study, we used the ensemble the four reanalysis datasets over their overlapping periods ${ }^{2}$ to analyze the atmospheric hydrological cycle over this region. The ensemble method further minimizes the uncertainties introduced by the choice of reanalysis datasets (Zhou and Yu 2005; Feng et al. 2012; Li et al. 2013b; Lin et al. 2014).

The summer season is defined as June-July-August (JJA) and the seasonal mean refers to the average over JJA. The SE US is defined as the terrestrial domain over $91^{\circ} \mathrm{W}-$ $76^{\circ} \mathrm{W}, 25^{\circ} \mathrm{N}-36.5^{\circ} \mathrm{N}$, namely seven states including North Carolina, South Carolina, Georgia, Florida, Alabama, Tennessee and Mississippi. Precipitation and moisture transport are domain averaged to emphasize the large-scale $(>1,000 \mathrm{~km})$ features of the summertime hydroclimate over the SE US. We are aware of the spatial heterogeneity in SE US summer precipitation (e.g. Konard 1997; Rhee et al. 2008), which cannot be fully resolved by current reanalysis datasets and CMIP5 models. However, the variation of SE US summer precipitation can be largely explained by a spatially homogenous pattern ( $\mathrm{Li}$ et al. 2013b). This homogenous large-scale pattern is well captured by most CMIP5 models (Li et al. 2013a). Thus, both observational data and CMIP5 models show a high confidence in quantifying the area-averaged summertime hydroclimate over the SE US, ensuring the reliability of the analysis results.

Future changes in SE US summertime hydroclimate are investigated by analyzing the atmospheric hydrological processes simulated by CMIP5 models. Two experiments are considered: the Historical run (1950-1999) and the Representative Concentration Pathway (RCP) 4.5 scenario (2050-2099). The Historical experiments represent the current climate and are driven by observed atmospheric composition changes. The time-evolving land cover changes are for the first time considered in CMIP5 models (Taylor et al. 2012). The RCP4.5 is a midrange mitigation emission scenario, within which the $\mathrm{CO}_{2}$ concentration increases to $650 \mathrm{ppm}$ in 2100 and is stabilized afterward.

\footnotetext{
2 During 1958-1999, the atmospheric moisture budget is calculated as the average of NCEP/NCAR and ERA-40; during 1979-1999, the moisture budget is calculated as the average of NCEP/NCAR, ERA40, JRA-25 and NARR. From 1950 to 1957, only NCEP/NCAR dataset is used.
}

Table 1 CMIP5 models used in this study

\begin{tabular}{llcc}
\hline Model & Resolution (Lat. $\times$ Lon., & \multicolumn{2}{l}{ Ensemble members } \\
\cline { 3 - 4 } & & Historical & RCP4.5 \\
\hline BCC-CSM1-1 & T42L26 & 3 & 1 \\
CCSM4 & $192 \times 228$ L26 & 6 & 6 \\
CESM1-BGC & $192 \times 228$ L26 & 1 & 1 \\
CESM1-WACCM & $96 \times 144$ L26 & 4 & 3 \\
CNRM-CM5 & T127L32 & 10 & 1 \\
CSIRO-Mk3.6.0 & T63L18 & 10 & 10 \\
FGOALS-s2 & $128 \times 108$ L26 & 3 & 2 \\
GFDL-CM3 & $90 \times 144$ L48 & 3 & 1 \\
GFDL-ESM2G & $90 \times 144$ L24 & 3 & 1 \\
GFDL-ESM2M & $90 \times 144$ L24 & 3 & 1 \\
HadGEM2-CC & $144 \times 192 L 60$ & 3 & 1 \\
INM-CM4 & $120 \times 180 L 21$ & 1 & 1 \\
IPSL-CM5A-LR & $95 \times 96 L 39$ & 5 & 4 \\
MIROC5 & T85L40 & 4 & 3 \\
MIROC-ESM & T42L80 & 3 & 1 \\
MPI-ESM-LR & T63L47 & 3 & 3 \\
MRI-CGCM3 & T159L48 & 5 & 1 \\
NorESM1-M & $96 \times 144 L 26$ & 2 & 1 \\
\hline
\end{tabular}

Meanwhile, the radiative forcing steadily increases to $4.5 \mathrm{~W} \mathrm{~m}^{-2}$ till 2100 and is then stabilized (Moss et al. 2010; Taylor et al. 2012). These two experiments are the core of CMIP5 long-term experiments, which are given higher priority by modeling centers (Taylor et al. 2009, 2012). Thus, output from these two experiments consists of larger sample size and ensures the statistical robustness of the analysis. We analyze 18 models that provide all variables required for the analysis of atmospheric hydrological cycle (Table 1).

\section{Variance partition algorithm and its application to diagnose hydroclimatic variance change}

The analysis of the SE US summertime hydrological cycle in the current climate indicates that the variance of SE US summer precipitation can largely be explained by the seasonal mean component of moisture transport (MTM) (Li et al. 2013b; see also "Appendix 1"). The MTM variance, in turn, is determined by the combined contributions from thermodynamic (specific humidity) and dynamic (wind) factors. However, quantification of thermodynamic and dynamic contributions to MTM variance is difficult, because sample variance reflects the second moment of the statistical samples, which is intrinsically more complicated than the sample mean. Specifically, the changes of MTM variance are not only caused by the changes in the variance 
of thermodynamic and dynamic factors, but also by the changes in the mean states of thermodynamics and dynamics, as well as their covariance. The multiple contributing factors make it hard to diagnose the processes/ factors key to the variance change. Previous studies on the partition of hydroclimatic variance change are based on strict assumptions, including that ENSO explains the majority of the variance of hydrological cycle (Seager et al. 2012). For seasons or regions where ENSO's influence is weak, the assumption will be violated and the variance change mechanism cannot be easily diagnosed.

This study develops an algorithm, which requires no prior assumptions, to explicitly quantify the thermodynamic and dynamic contributions to variance changes of regional hydrological cycle. According to the definition of sample variance: $\operatorname{var}(\mathbf{Y})=\frac{1}{n-1} \sum_{i=1}^{n}\left(y_{i}-\frac{1}{n} \sum_{i=1}^{n} y_{i}\right)^{2}$, no information regarding data alignment is needed to calculate sample variance (Hoff 2009; DeGroot and Schervish 2011). Utilizing such a statistical property, the algorithm is formulated as following.

Let $\mathbf{Y}_{\mathbf{1}}$ denote a n-year such as 50-year MTM time series in climate state 1 , and $\mathbf{Y}_{\mathbf{2}}$ in climate state 2 (i.e., $\mathbf{Y}_{\mathbf{1}}$ and $\mathbf{Y}_{\mathbf{2}}$ are column vectors).

Step (1) Permute both $\mathbf{Y}_{\mathbf{1}}$ and $\mathbf{Y}_{\mathbf{2}}$ so that $y_{\pi \_1} \leq y_{\pi \_2} \leq \ldots \leq y_{\pi \_i} \ldots \leq \ldots \leq y_{\pi \_50}$. Here, the subscript $\pi$ denotes the time series after permutation, while the number $i \in\{1,2, \ldots, 50\}$ in the subscript denotes the $\mathrm{i}$-th element of the time series. After the permutation, the year with the strongest moisture convergence is ranked the first, while that with the strongest moisture divergence is ranked the last. The sample variance calculated from the permutated time series remains the same as that from the original time series without permutation. However, the quantification of thermodynamic and dynamic contributions to the variance changes can be simplified (See below and “Appendix 2").

Step (2) Identify specific humidity (thermodynamics) and wind (dynamics) corresponding to each MTM quantile in climate state 1 and 2: $\mathbf{Y}_{\mathbf{1} \pi}=\int_{0}^{p_{s}} \nabla \cdot\left(q_{1 \pi} \vec{V}_{1 \pi}\right) d p$ and $\mathbf{Y}_{2 \pi}=\int_{0}^{p_{s}} \nabla \cdot\left(q_{2 \pi} \vec{V}_{2 \pi}\right) d p$ are column vectors with 50 elements (i.e. samples from the 50 -year time series). The ith element corresponding to the i-th quantile of the MTM are $y_{1 \pi \_i}=\int_{0}^{p_{s}} \nabla \cdot\left(q_{1 \pi \_i} \vec{V}_{1 \pi \_i}\right) d p, y_{2 \pi \_i}=\int_{0}^{p_{s}} \nabla$. $\left(q_{2 \pi \_i} \vec{V}_{2 \pi \_i}\right) d p$, respectively.

Step (3) Calculate the quantile differences of specific humidity (thermodynamics) and wind (dynamics) between climate state 1 and state 2: $\Delta q_{\pi}=q_{2 \pi}-q_{1 \pi} ; \Delta \vec{V}_{\pi}=$ $\vec{V}_{2 \pi}-\vec{V}_{1 \pi}$, with the changes of the thermodynamic and dynamic components in the i-th quantile being $\Delta q_{\pi_{-} i}=$ $q_{2 \pi \_i}-q_{1 \pi \_i}$ and $\Delta \vec{V}_{\pi \_i}=\vec{V}_{2 \pi \_i}-\vec{V}_{1 \pi \_i}$, respectively.

The variance of MTM in state 2 can be expressed as:

$$
\begin{aligned}
\operatorname{var}\left(\mathbf{Y}_{\mathbf{2} \pi}\right)= & \operatorname{var}\left(\int_{0}^{p_{s}} \nabla \cdot\left(q_{1 \pi} \vec{V}_{1 \pi}\right) d p+\int_{0}^{p_{s}} \nabla \cdot\left(\Delta q_{\pi} \vec{V}_{1 \pi}\right) d p\right. \\
& \left.+\int_{0}^{p_{s}} \nabla \cdot\left(q_{1 \pi} \Delta \vec{V}_{\pi}\right) d p+\int_{0}^{p_{s}} \nabla \cdot\left(\Delta q_{\pi} \Delta \vec{V}_{1 \pi}\right) d p\right)
\end{aligned}
$$

Generally, the $\Delta q_{\pi}$ and $\Delta \vec{V}_{\pi}$ are small deviations from $q_{1 \pi}$ and $\vec{V}_{1 \pi}$, after the permutation. ${ }^{3}$ Thus the term $\int_{0}^{p_{s}} \nabla \cdot\left(\Delta q_{\pi} \Delta \vec{V}_{\pi}\right) d p$ can be neglected, and Eq. 1 can be simplified as:

$$
\begin{aligned}
\operatorname{var}\left(\mathbf{Y}_{2 \pi}\right) \cong \operatorname{var} & \left(\int_{0}^{p_{s}} \nabla \cdot\left(q_{1 \pi} \vec{V}_{1 \pi}\right) d p+\int_{0}^{p_{s}} \nabla \cdot\left(\Delta q_{\pi} \vec{V}_{1 \pi}\right) d p\right. \\
& \left.+\int_{0}^{p_{s}} \nabla \cdot\left(q_{1 \pi} \Delta \vec{V}_{\pi}\right) d p\right)
\end{aligned}
$$

Step (4) Partition Eq. 2 using the statistical relationship that $\operatorname{var}(a+b+c)=\operatorname{var}(a)+\operatorname{var}(b)+\operatorname{var}(c)+$ $2 \operatorname{cov}(a, b)+2 \operatorname{cov}(a, c)+2 \operatorname{cov}(b, c)$, where $\operatorname{cov}(a, b)$ is the covariance operator:

$$
\begin{aligned}
\operatorname{var} & \left(\int_{0}^{p_{s}} \nabla \cdot\left(q_{1 \pi} \vec{V}_{1 \pi}\right) d p+\int_{0}^{p_{s}} \nabla \cdot\left(\Delta q_{\pi} \vec{V}_{1 \pi}\right) d p\right. \\
& \left.+\int_{0}^{p_{s}} \nabla \cdot\left(q_{1 \pi} \Delta \vec{V}_{\pi}\right) d p\right) \\
= & \operatorname{var}\left(\int_{0}^{p_{s}} \nabla \cdot\left(q_{1 \pi} \vec{V}_{1 \pi}\right) d p\right)+\operatorname{var}\left(\int_{0}^{p_{s}} \nabla \cdot\left(\Delta q_{\pi} \vec{V}_{1 \pi}\right) d p\right) \\
& +\operatorname{var}\left(\int_{0}^{p_{s}} \nabla \cdot\left(q_{1 \pi} \Delta \vec{V}_{\pi}\right) d p\right) \\
& +2 \operatorname{cov}\left(\int_{0}^{p_{s}} \nabla \cdot\left(q_{1 \pi} \vec{V}_{1 \pi}\right) d p, \int_{0}^{p_{s}} \nabla \cdot\left(\Delta q_{\pi} \vec{V}_{1 \pi}\right) d p\right) \\
& +2 \operatorname{cov}\left(\int_{0}^{p_{s}} \nabla \cdot\left(q_{1 \pi} \vec{V}_{1 \pi}\right) d p, \int_{0}^{p_{s}} \nabla \cdot\left(q_{1 \pi} \Delta \vec{V}_{\pi}\right) d p\right) \\
& +2 \operatorname{cov}\left(\int_{0}^{p_{s}} \nabla \cdot\left(\Delta q_{\pi} \vec{V}_{1 \pi}\right) d p, \int_{0}^{p_{s}} \nabla \cdot\left(q_{1 \pi} \Delta \vec{V}_{\pi}\right) d p\right)
\end{aligned}
$$

\footnotetext{
${ }^{3}$ Before permutation, $\operatorname{var}\left(\int_{0}^{p_{s}} \nabla \cdot(\Delta q \Delta \vec{V}) d p\right)$ cannot be neglected, because, due to the randomness of climate variability, $\Delta q$ and $\Delta \vec{V}$ is not necessarily a small deviation from the original climate state. Thus, permutation is needed to separate the thermodynamic and dynamic contributions in the covariance terms (see "Appendix 2").
} 
In the right-hand-side of Eq. 3, $\operatorname{var}\left(\int_{0}^{p_{s}} \nabla \cdot\left(q_{1 \pi} \vec{V}_{1 \pi}\right) d p\right)$ $=\operatorname{var}\left(\mathbf{Y}_{1 \pi}\right)$, thus,

$$
\begin{aligned}
& \operatorname{var}\left(\mathbf{Y}_{2 \pi}\right)-\operatorname{var}\left(\mathbf{Y}_{1 \pi}\right) \\
& \cong \underbrace{\operatorname{var}\left(\int_{0}^{p_{s}} \nabla \cdot\left(\Delta q_{\pi} \vec{V}_{1 \pi}\right) d p\right)}_{A} \\
& +\underbrace{2 \operatorname{cov}\left(\int_{0}^{p_{s}} \nabla \cdot\left(q_{1 \pi} \vec{V}_{1 \pi}\right) d p, \int_{0}^{p_{s}} \nabla \cdot\left(\Delta q_{\pi} \vec{V}_{1 \pi}\right) d p\right)}_{B} \\
& +\underbrace{\operatorname{var}\left(\int_{0}^{p_{s}} \nabla \cdot\left(q_{1 \pi} \Delta \vec{V}_{1 \pi}\right) d p\right)}_{C} \\
& +\underbrace{2 \operatorname{cov}\left(\int_{0}^{p_{s}} \nabla \cdot\left(q_{1 \pi} \vec{V}_{1 \pi}\right) d p, \int_{0}^{p_{s}} \nabla \cdot\left(q_{1 \pi} \Delta \vec{V}_{\pi}\right) d p\right)}_{D} \\
& +\underbrace{2 \operatorname{cov}\left(\int_{0}^{p_{s}} \nabla \cdot\left(\Delta q_{\pi} \vec{V}_{1 \pi}\right) d p, \int_{0}^{p_{s}} \nabla \cdot\left(q_{1 \pi} \Delta \vec{V}_{\pi}\right) d p\right)}_{E} .
\end{aligned}
$$

The left hand side of Eq. 4 is the differences of MTM variance between climate state 1 and state 2 . On the right hand side, the thermodynamic and dynamic contributions to variance changes are quantified explicitly. Specifically, terms A and $\mathrm{B}$ (terms $\mathrm{C}$ and $\mathrm{D}$ ) only involve the differences in specific humidity (wind), and thus reflect the thermodynamic (dynamic) contributions to variance changes. Term E involves both specific humidity and wind, and thus denotes the coupling between thermodynamic and dynamic processes.

Equation 4 partitions the difference in MTM variance between two climate states (such as the twentieth and twenty-first century climate) into those resulting from thermodynamic components (terms A and B), dynamic components (terms $\mathrm{C}$ and $\mathrm{D}$ ), and their coupling processes (term E). Thus, it can be implemented to diagnose thermodynamic and dynamic contributions to the changes in MTM variance in future climate. This algorithm can also be implemented to evaluate GCM simulations of MTM variance by comparing model simulations with the reanalysis ensemble.

It is noteworthy that this algorithm utilizes the general rule of sample variance calculation, and requires no additional assumptions about statistical samples. Thus, the algorithm can be applied to any region and/or any season to diagnose the variance changes in regional hydroclimate. In this study, this algorithm is applied to SE US summertime hydroclimate to (1) provide a process-level evaluation of CMIP5 model skill in simulating the variance of SE US summer precipitation, and (2) quantify the thermodynamic and dynamic contributions to MTM and precipitation variance change in a warming climate in order to understand the mechanisms responsible for future changes in hydroclimatic variance.

\section{Evaluation of SE US summertime hydroclimatic processes in CMIP5 models}

The analysis of observed atmospheric hydrological cycle over the Southeast suggests that the MTM process is essential to SE US summer precipitation and its variance (Fig. 6). Thus, it is likely that the simulations of the MTM processes also determine the skill of the "state-of-the-art" GCMs in simulating SE US summer precipitation variance. Hereafter we will evaluate the performance of CMIP5 models in simulating summer precipitation variance and its relationship with the MTM. Causes of the modeled biases in SE US summer precipitation variance will also be diagnosed using the variance partition algorithm formulated in Sect. 3.

\subsection{CMIP5 model simulations of SE US summer precipitation variance and its relationship with the MTM}

The observed precipitation and the reanalysis ensemble suggest that the maximum likelihood estimator (MLE) of precipitation and MTM variance is 0.42 and $0.49 \mathrm{~mm}^{2}$ day $^{-2}$, and the $99 \%$ confidence intervals (CIs) are [0.22 $0.67]$ and [0.32 0.84] $\mathrm{mm}^{2} \mathrm{day}^{-2}$, respectively (Fig. 1a). These statistics provide an evaluation metric to assess CMIP5 model simulations of SE US summertime hydroclimate. The precipitation and MTM variance in the ensemble of CMIP5 models are within the $99 \% \mathrm{CI}$ of the observations, although the ensemble of the climate models underestimates the variance of both variables compared to the MLE of observations (Fig. 1a). Generally, the simulated precipitation and MTM variance shows a linear relationship, with models simulating higher MTM variance also simulating higher precipitation variance. It thus indicates the importance of MTM for a reasonable representation of SE US summertime hydroclimatic variance (Fig. 1a). Among the 18 CMIP5 models, seven models reasonably capture the precipitation and MTM variance, i.e., the simulated variances of both variables are within the $99 \%$ CI derived from observations (Fig. 1a). Furthermore, six out of the seven models capture the observed 


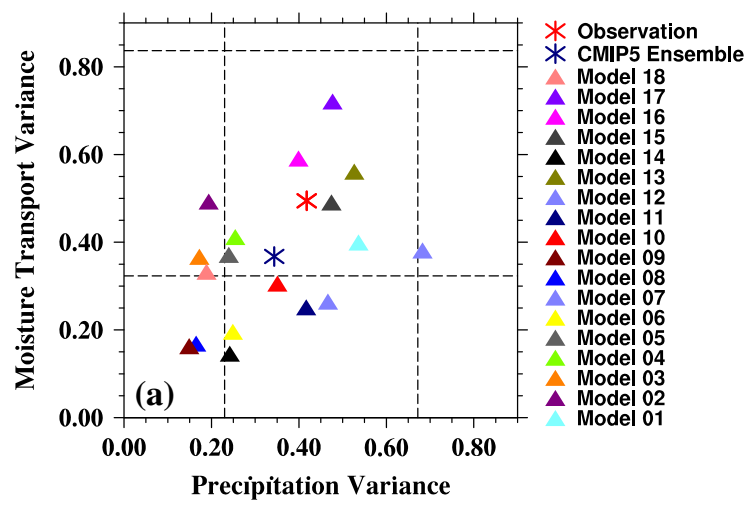

Fig. 1 a Variance of SE US summer precipitation (unit: $\mathrm{mm}^{2}$ day ${ }^{-2}$ ) and MTM (unit: $\mathrm{mm}^{2} \mathrm{day}^{-2}$ ) in observations (red asterisk), the simulations by CMIP5 models (up triangles), and the ensemble of CMIP5 models (blue asterisk). The black dashed lines are the lower and upper and bounds of $99 \%$ confidence interval (CI) of observed precipitation and MTM variance; $\mathbf{b}$ correlation between precipitation and MTM versus the variance of MTM in observations (red asterisk),

relationship between precipitation and moisture transport (Fig. 1b), where the simulated MTM explains more than $50 \%$ of the precipitation variance. These six models are categorized as 1 st class models due to their capability to represent the key processes responsible for SE US summer precipitation variance.

The reasonable simulations of SE US summertime hydroclimatic processes by the six models are attributable to their relatively higher variance of the MTM. As shown in Fig. 1b, models that simulate higher MTM variance tend to have higher correlation coefficients between the simulated MTM and precipitation. The result is verified by observation-based Monte-Carlo samples. 5000 Monte-Carlo sample sets are created, each consisting of two 30-year hypothetical time series that are randomly drawn from the observed precipitation and MTM, respectively. The correlation coefficients between precipitation and MTM are calculated and are shown against the MTM variance in Fig. 1b. It is shown that the relationship between MTM and precipitation depends on the variance of MTM. Generally, the sample sets with smaller MTM variance show less significant correlation coefficients between MTM and summer precipitation (Fig. 1b). The correlation coefficients increase as the MTM variance increases, indicating that MTM has a stronger modulation effect on SE US summer precipitation at its high variance state (Fig. 1b). Such an observed empirical relationship can also be seen in the simulations by CMIP5 models (Fig. 1b), which further emphasizes the importance of MTM variance in reasonable simulations of SE US summertime hydroclimate and its variance.

In summary, evaluation of CMIP5 models indicates that six out of $18(33 \%)$ models reasonably capture the variance of SE US summer precipitation at process level

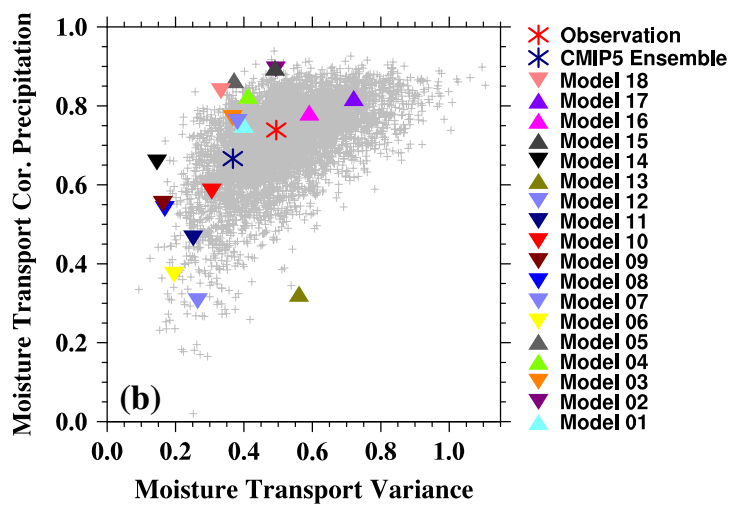

the simulations by CMIP5 models (triangles), and the ensemble of CMIP5 models (blue asterisk). The up triangles represent the models that simulate both the variance of precipitation and MTM within the observed $99 \% \mathrm{CI}$; while the down triangles denote the simulations outside the $99 \%$ CI. In b, the results using the 5,000 hypothetical time series with samples randomly drawn from the observed summer precipitation and MTM are shown in gray crosses

(Fig. 1). These models are ranked as 1 st class models and will be implemented to project future changes in SE US summer precipitation, and to analyze the mechanisms underlying the future changes in precipitation.

\subsection{Causes of the models bias in the MTM variance}

Observations demonstrate that the MTM is important to precipitation variance over the SE US (Fig. 6). In CMIP5 models, the variance of MTM statistically determines the skills in simulating the relationship between MTM and precipitation (Fig. 1b), i.e., the most important hydrological relationship for SE US summer precipitation variance (Li et al. 2013b; see also Fig. 6). Thus, realistically simulating the variance of MTM is vital to capture the SE US summertime hydroclimate. However, most $(83.3 \%)$ CMIP5 models underestimate the variance of MTM compared to the MLE of the reanalysis ensemble, and the bias in multi-model ensemble is $-0.12 \mathrm{~mm}^{2}$ day $^{-2}$ (Fig. 1a). It indicates that summer precipitation extremes tend to be underestimated by CMIP5 models in the Southeast. Understanding the factors that cause the underestimation of MTM variance, either thermodynamically or dynamically, will help to improve the skills of GCMs in simulating summer precipitation variance and extremes in the region.

The variance partition algorithm in Sect. 3 is implemented to quantify the MTM bias introduced by errors in CMIP5 model-simulated thermodynamic and dynamic factors. Here, $q_{1 \pi}\left(q_{2 \pi}\right)$ and $\vec{V}_{1 \pi}\left(\vec{V}_{2 \pi}\right)$ are the specific humidity and wind in the reanalysis ensemble (each CMIP5 model) corresponding to each quantile of MTM. $\Delta q_{\pi}=q_{2 \pi}-q_{1 \pi} \quad$ and $\quad \Delta \vec{V}_{\pi}=\vec{V}_{2 \pi}-\vec{V}_{1 \pi}$ denote the 


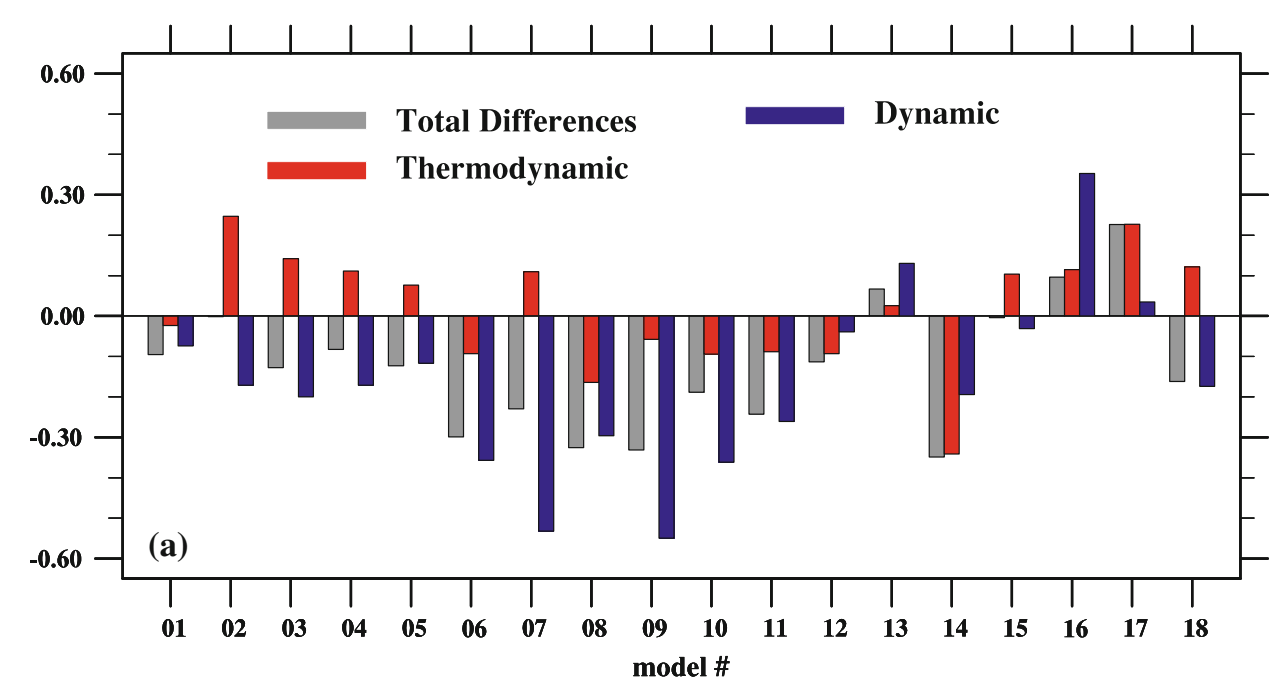

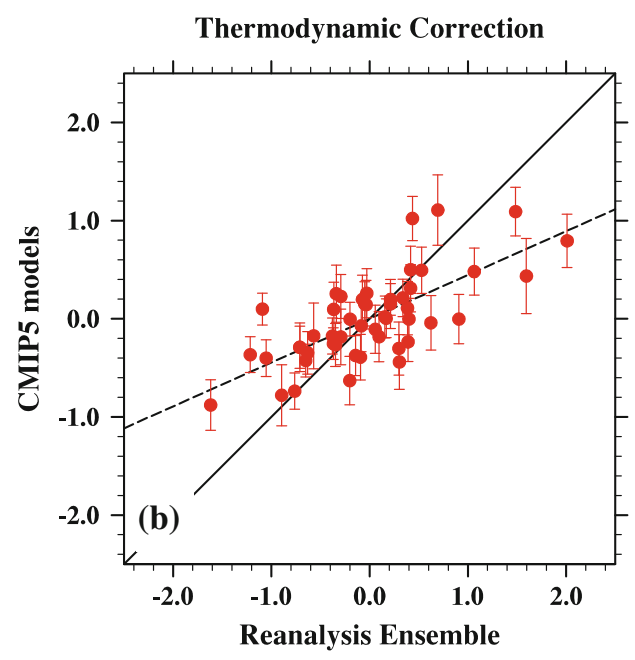

Fig. 2 a Bias in the MTM variance as simulated by 18 CMIP5 models (gray bars, unit: $\mathrm{mm}^{2} \mathrm{day}^{-2}$ ), and the thermodynamic (red bars, calculated as terms A and B in Eq. 4) and dynamic (blue bars, calculated as terms C and D in Eq. 4) contributions to the bias in MTM variance (unit: $\mathrm{mm}^{2} \mathrm{day}^{-2}$ ); b, c are the quantile-quantile (QQ) plots of thermodynamically and dynamically corrected MTM in

quantile differences in thermodynamic and dynamic components between CMIP5 models and the reanalysis ensemble.

The thermodynamic (dynamic) contributions are quantified as the sum of Terms A and B (Terms C and D) in Eq. 4, and are compared with the total MTM variance bias in Fig. 2a. In 14 out of the 18 (77.8 \%) CMIP5 models, the biases in MTM variance are likely caused by the dynamic components. In contrast, thermodynamic components are only important in four $(22.2 \%$ ) models (Fig. 2a; model \#12, \#14, \#16, and \#17). Averaged over the 18 CMIP5 models, the errors in dynamic components contribute $-0.14 \mathrm{~mm}^{2}$ day $^{-2}$ bias to the variance of MTM, whereas the thermodynamic components only contribute $0.01 \mathrm{~mm}^{2}$ day $^{-2}$ bias. The bias caused by the dynamic

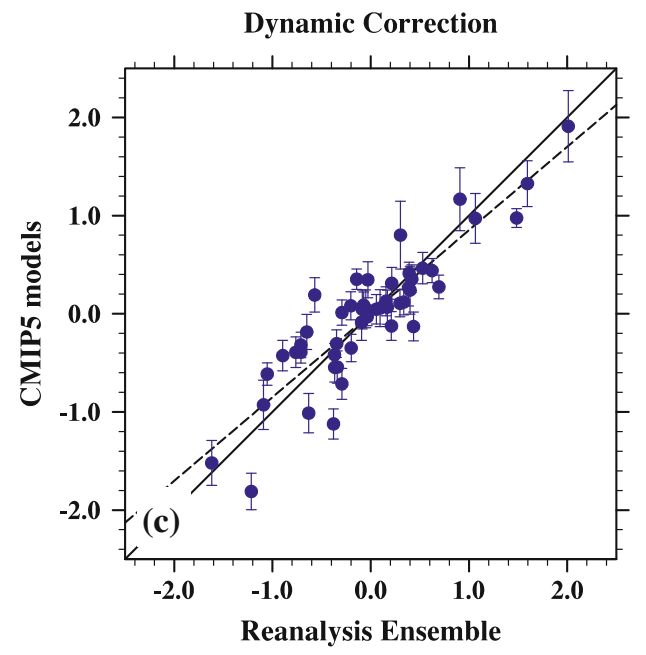

CMIP5 models versus the MTM calculated using reanalysis ensemble, respectively. The red and blue dots in $\mathbf{b}$ and $\mathbf{c}$ are the ensemble of CMIP5 models at each quantile, and the error bars denote the $95 \%$ CIs of the moisture transport derived from the 18 CMIP5 models. The solid and dashed lines in $\mathbf{b}$ and $\mathbf{c}$ are the one-to-one lines and the best least square fitting lines, respectively

components is close to the total MTM bias $\left(-0.12 \mathrm{~mm}^{2}\right.$ $\mathrm{day}^{-2}$ ) as in the ensemble of CMIP5 models. Comparatively, the dynamic components are more responsible for the underestimation of MTM variance than the thermodynamic components, according to the variance partition algorithm.

The contribution of dynamic components to the model bias in MTM variance is further verified by the quantilequantile (Q-Q) plots (Fig. 2b, c). In Fig. 2b, c, thermodynamic and dynamic components simulated by CMIP5 models are corrected to the reanalysis ensemble, respectively, at each MTM quantile. Merely correcting the model simulated thermodynamics (specific humidity), the MTM in the lower and upper quantiles are still underestimated compared to the reanalysis ensemble (Fig. 2b). The 


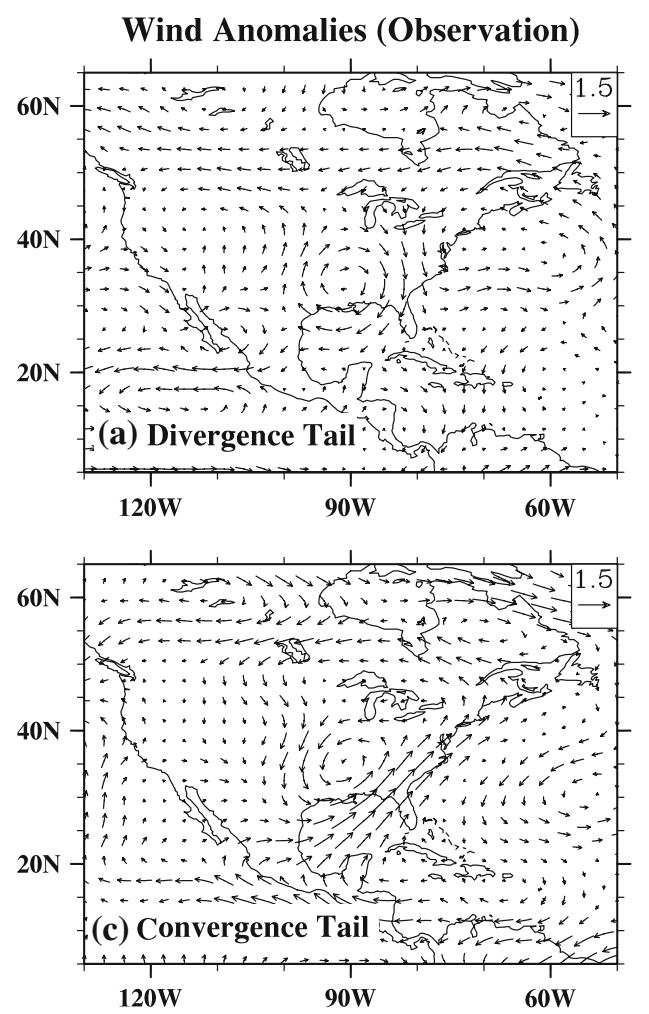

Fig. $3850 \mathrm{hPa}$ horizontal wind anomalies (vector, unit: $\mathrm{m} \mathrm{s}^{-1}$ ) associated with the upper $(\mathbf{a}, \mathbf{b})$ and lower $(\mathbf{c}, \mathbf{d})$ 10-percentile of MTM in observations (left panels) and the ensemble of CMIP5

limited improvement brought by thermodynamic corrections indicates that the MTM bias more likely comes from the errors in the dynamic fields, i.e., atmospheric circulation. After correcting the circulation fields, the probability behavior of MTM better approximates that of the reanalysis ensemble, especially at the upper and lower quantiles (Fig. 2c).

Furthermore, the modeled dynamic components at the upper and lower 10-percentile of MTM, i.e., highest moisture convergence and divergence, are compared to those in the reanalysis ensemble using the $850 \mathrm{hPa}$ wind anomalies (Fig. 3). Corresponding to the upper (lower) 10-percentile of MTM, the reanalysis ensemble shows an anomalous anticyclone (cyclone) over the SE US (Fig. 3a, c). In the ensemble of CMIP5 models (Fig. 3b, d), both the anticyclone and cyclone associated with the upper and lower tail of MTM are substantially underestimated compared to the reanalysis ensemble (Fig. 3a, b). Such a bias in CMIP5 model simulated dynamic fields may result from the incapability of most CMIP5 models to simulate the modulation effects of the North Atlantic Subtropical High (NASH) western ridge on SE US summer precipitation ( $\mathrm{Li}$ et al. 2013a). The weaker circulation anomalies in CMIP5 model simulations lead to insufficient
Wind Anomalies (Model Ensemble)
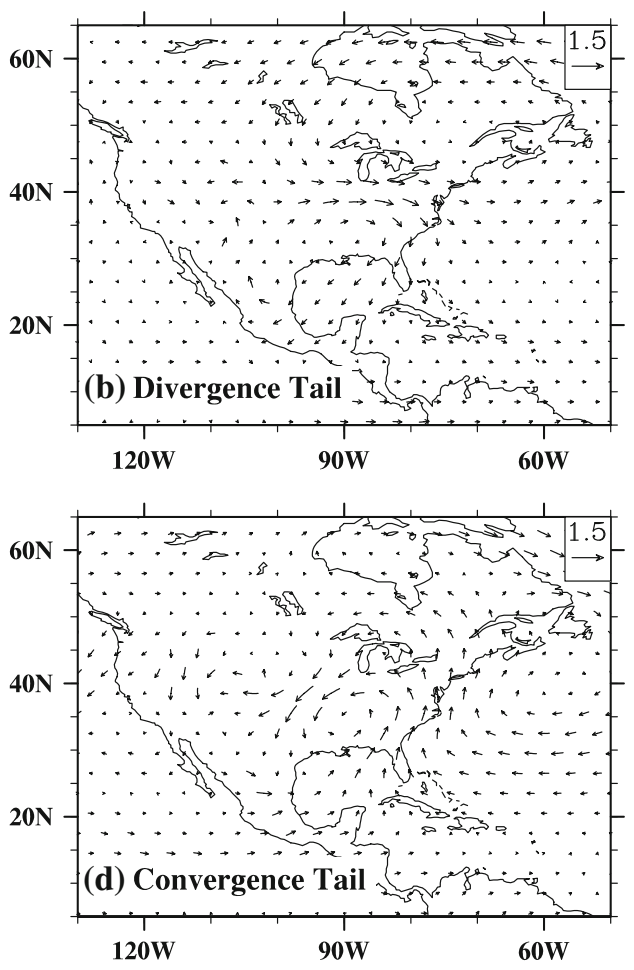

models (right panels). Here, the upper (lower) 10-percentile of MTM corresponding to moisture divergence (convergence)

convergence/divergence of moisture over the SE US, thus weakening the MTM and precipitation variance in CMIP5 models.

In conclusion, CMIP5 models generally underestimate the MTM variance over the SE US, which further leads to an underestimation of precipitation variance. The variance partition algorithm identifies that the dynamic components are the primary sources of the model biases in simulating the MTM variance. Thus, improvements in the dynamic components are needed to improve the prediction skills of CMIP5 models in simulating the variance of SE US summertime hydroclimate.

\section{Thermodynamic and dynamic contributions to the intensified variance of SE US summer precipitation in a warming climate}

Compared to observations, six out of the 18 (33\%) CMIP5 models reasonably capture the variance of SE US summer precipitation and MTM, as well as the relationship between the precipitation and MTM. These six models are further analyzed to study future changes in SE US summertime hydroclimate. 

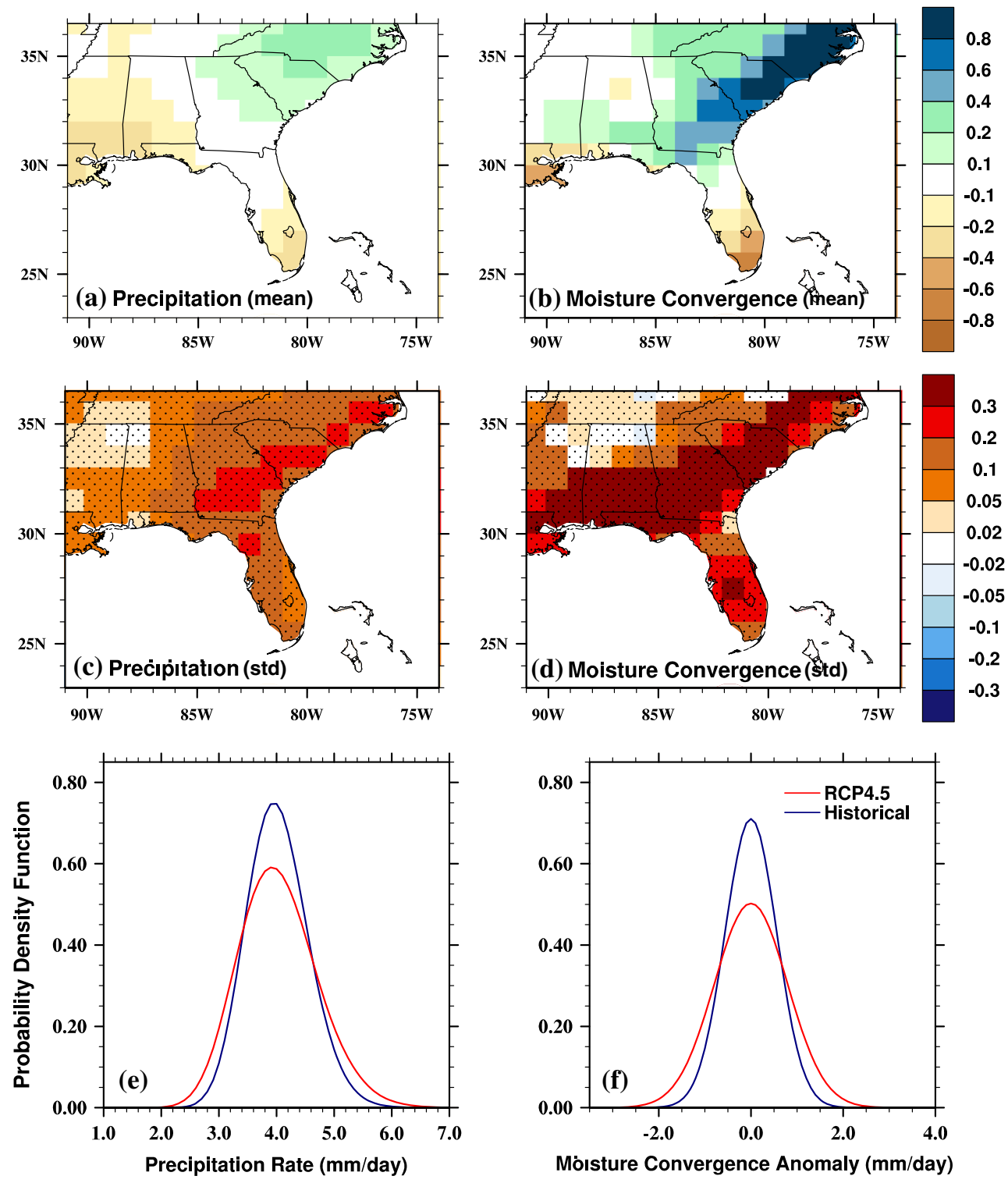

Fig. 4 Changes in the SE US summertime climatology of a precipitation and b MTM (shaded, units: $\mathrm{mm} \mathrm{day}^{-1}$ ); and the standard deviation of $\mathbf{c}$ precipitation and $\mathbf{d}$ MTM (shaded, units: $\mathrm{mm}$ day $^{-1}$ ) from historical run (1950-1999) to RCP4.5 scenarios (2050-2099). The probability density function (PDF) curves constructed based on

areal-averaged SE US summer precipitation and MTM anomalies are shown in $\mathbf{e}$ and $\mathbf{f}$ respectively. In $\mathbf{a}$ and $\mathbf{b}$, the grid points with changes significant at 0.05 level by $t$ test are stippled; while in $\mathbf{c}$ and $\mathbf{d}$ the stippled are the grid points with variance changes passing $\alpha=0.05 \mathrm{~F}$ test

\subsection{Future changes in SE US summertime hydroclimate}

Figure 4 shows the projection of future changes in SE US summer precipitation and MTM by the ensemble of the 1st class models. Changes in precipitation and MTM climatology show a dipole pattern, with precipitation and moisture convergence increasing over the eastern coasts, but decreasing westward (Fig. 4a, b). Over the SE US, the changes in mean precipitation and MTM as projected by

the ensemble of 1st class models are too weak to pass the student $t$ test (Fig. 4a, b), consistent with (Kitoh et al. 2013). In contrast, the 1 st class models consistently project a significant increase in summer precipitation variance ( $\alpha=0.05$ level by $F$ test) throughout the entire domain (Fig. 4c). The increases in precipitation variance are most apparent along the eastern coast, slightly weak inland. The increase in precipitation variance and its spatial pattern are consistent with the projected MTM variance throughout the entire domain (Fig. 4d). Such a consistency suggests that 

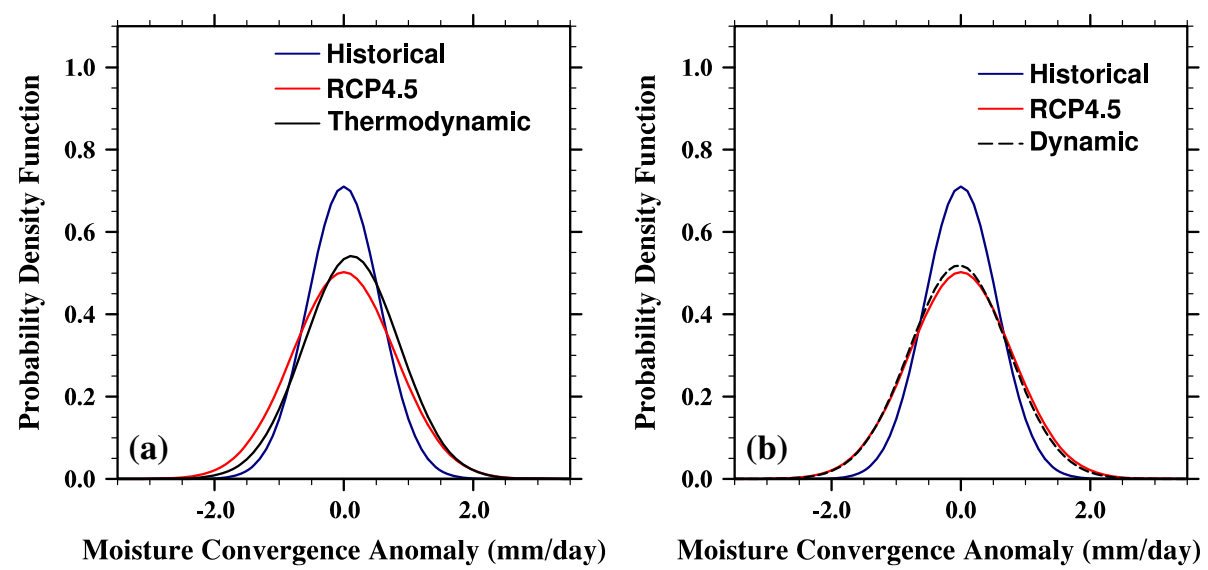

Fig. 5 PDF curves of a thermodynamic (solid black curve) and $\mathbf{b}$ dynamic components (dashed black curve) of MTM over the SE US and their comparison with the historical (solid blue curve) and RCP4.5 PDF (solid red curve)

the changes in MTM processes might be responsible for the intensified precipitation variance over the SE US in a warming climate.

Future changes in SE US summer precipitation and MTM can also be illustrated by the probability density functions (PDFs) of the areal-averaged precipitation and MTM. The Normal distribution is used to fit the MTM, while the Gamma distribution is used to fit the summer precipitation considering the non-negative constraint and the skewness of precipitation distribution. ${ }^{4}$ Prior to constructing the PDFs, both precipitation and MTM samples are quantile normalized to avoid artificial variance introduced by the spread of model response to greenhouse gases (GHGs) forcing (Bolstad et al. 2003; Li et al. 2013a).

The precipitation PDFs show no apparent shift in the mean under the RCP4.5 scenarios, whereas the variance increases significantly (Fig. 4e), since the scale parameters (reflecting the "statistical dispersion") increase at $\alpha=0.01$ level. Both the dry and wet tails of the RCP4.5 PDF extend further, suggesting an intensification of precipitation variance in a warming climate (Fig. 4e), consistent with $\mathrm{Li}$ et al. (2011, 2013a).

The increases in SE US summer precipitation variance can be explained by the intensified variance in MTM (Fig. 4f). Compared to the Historical simulations, increases in GHGs do not shift the modes of MTM PDFs at all, indicating the climatology of MTM will likely remain the same in the future in the region (Fig. 4f). In contrast, the

\footnotetext{
4 The Log-Normal, Log-Pearson Type III, and Generalized extreme value-Type II distribution kernels have also been tested. For SE US summer precipitation, these distribution kernels do not show obvious advantage over the Gamma kernel, although they imply higher complexity. Most importantly, the precipitation PDFs constructed using these different kernels suggest the same changes in future precipitation over the SE US. Thus, only the results based on Gamma distribution kernel are discussed and presented here.
}

tails of the RCP4.5 PDF extend, suggesting both the divergence and convergence of moisture flux will be enhanced in the future (Fig. 4f). The intensified moisture divergence (convergence) over the SE US could lead to a deficit (excessiveness) in SE US summer precipitation, exacerbating drought (fluvial) conditions in a warming climate.

The similarities in the precipitation and MTM PDFs further emphasize the importance of MTM in regulating SE US summer precipitation variance in current and future climate. Overall, Fig. 4 suggests that the variance of SE US summer precipitation would further intensify in the future (Fig. 4c, e), which can be largely explained by the changes in MTM (Fig. 4d, f).

5.2 Thermodynamic and dynamic contributions to the intensification of SE US summer precipitation variance in the future

The 1st class models project that SE US summertime hydrological cycle will become more variable under the RCP4.5 scenarios. The variance of precipitation will significantly increase in a warming climate, which is tightly associated with an increased MTM variance. Previous studies suggest that both thermodynamics and dynamics can cause changes in hydrological cycle under warming scenarios (e.g., Held and Soden 2006; Huntington 2006; Vecchi et al. 2006; O'Gorman and Schneiderb 2009; Seager et al. 2010; Trenberth 2011). The thermodynamic and dynamic contributions to the intensified variance in SE US summer precipitation are assessed by applying the variance partition algorithm (Sect. 3) to the MTM.

For this application, $q_{2 \pi}\left(q_{1 \pi}\right)$ and $\vec{V}_{2 \pi}\left(\vec{V}_{1 \pi}\right)$ are the model simulated specific humidity and wind field under RCP4.5 scenarios (Historical runs) at each MTM quantile. $\Delta q_{\pi}=q_{2 \pi}-q_{1 \pi}$ and $\Delta \vec{V}_{\pi}=\vec{V}_{2 \pi}-\vec{V}_{1 \pi}$ represent the 


\section{SE US JJA moisture budget}
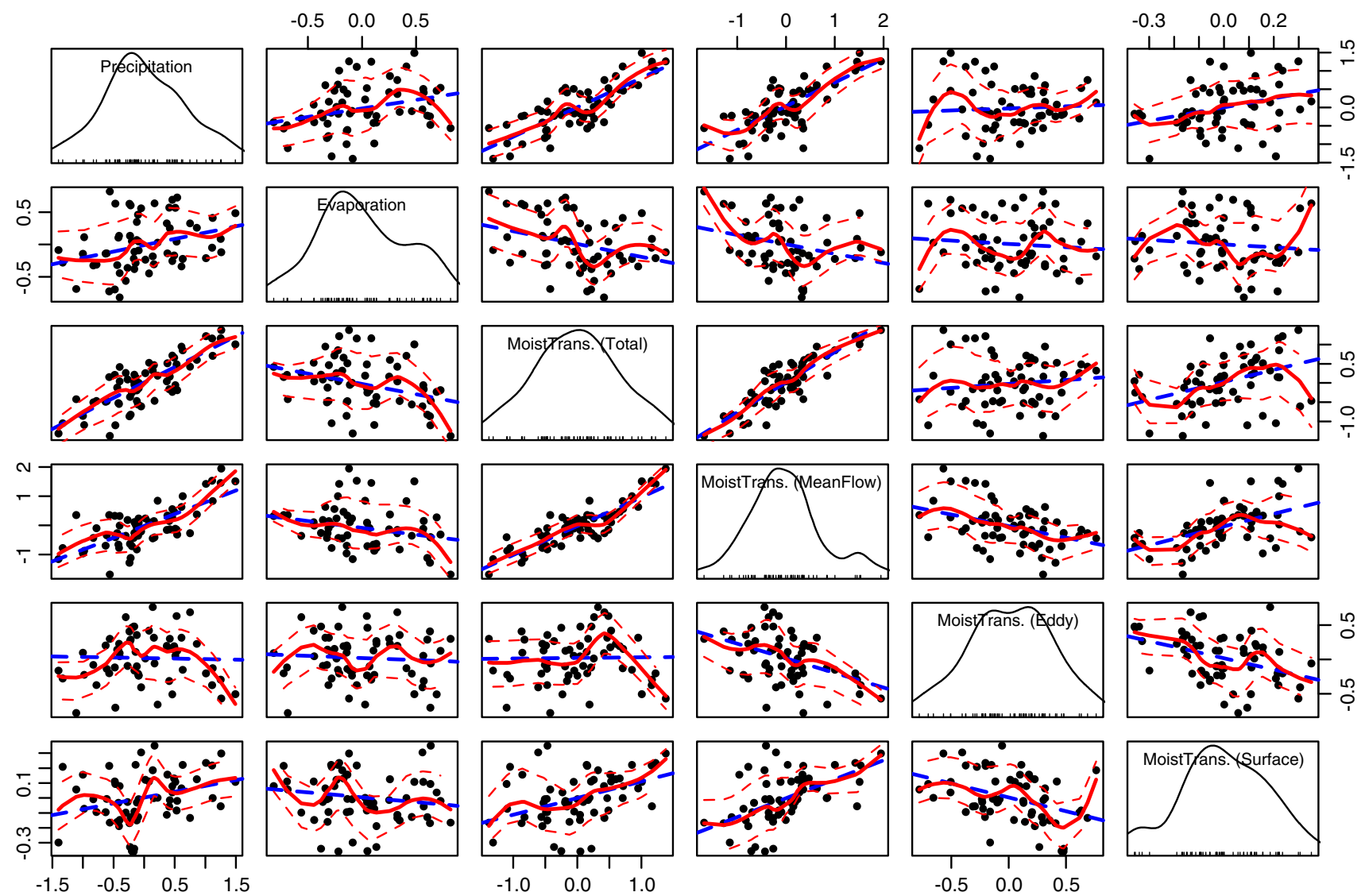

Fig. 6 Pairs plot showing the SE US summertime (JJA) moisture budget in the 1948-2007. The diagonal panel plots are the empirical PDFs constructed based on 60 data samples corresponding to the six components in moisture budget (Eqs. 7-8). The off diagonal panels show the scatter plot between the paired moisture budget

components. The solid red curves are the locally weighted scatterplot smoothing (LOWESS) curves fitted to the data samples. The dashed red curves represent the upper and lower bounds of the $95 \%$ confidence interval of the fitted LOWESS curve. The dashed blue lines are the best least square fitting lines

quantile-based thermodynamic and dynamic differences between RCP4.5 scenarios and Historical runs, respectively. The thermodynamic contributions to future changes in the MTM variance can be assessed by fixing $\Delta \vec{V}_{\pi}=0$; i.e., the dynamic components are kept at Historical level and do not contribute to variance changes in RCP4.5 MTM. Equation 4 demonstrates that changes in MTM variance come from Terms A and $\mathrm{B}$. This thermodynamically determined MTM variance can be expressed as:

$$
\begin{aligned}
\operatorname{var}\left(\mathbf{Y}_{\text {thm }}\right) \approx & \operatorname{var}\left(\mathbf{Y}_{\mathbf{1} \pi}\right)+\operatorname{var}\left(\int_{0}^{p_{s}} \nabla \cdot\left(\Delta q_{\pi} \vec{V}_{1 \pi}\right) d p\right) \\
& +2 \operatorname{cov}\left(\mathbf{Y}_{1 \pi}, \int_{0}^{p_{s}} \nabla \cdot\left(\Delta q_{\pi} \vec{V}_{1 \pi}\right) d p\right) \\
= & \operatorname{var}\left(\int_{0}^{p_{s}} \nabla \cdot\left(q_{2 \pi} \vec{V}_{1 \pi}\right) d p\right)
\end{aligned}
$$

Similarly, by fixing $\Delta q_{\pi}=0$, Terms $\mathrm{C}$, D, and $\mathrm{E}$ in Eq. 4 vanish, and the dynamic contributions to moisture transport variance can be expressed as:

$$
\begin{aligned}
\operatorname{var}\left(\mathbf{Y}_{\mathbf{d y n}}\right) \approx & \operatorname{var}\left(\mathbf{Y}_{\mathbf{1} \pi}\right)+\operatorname{var}\left(\int_{0}^{p_{s}} \nabla \cdot\left(q_{1 \pi} \Delta \vec{V}_{\pi}\right) d p\right) \\
& +2 \operatorname{cov}\left(\mathbf{Y}_{1 \pi}, \int_{0}^{p_{s}} \nabla \cdot\left(q_{1 \pi} \Delta \vec{V}_{\pi}\right) d p\right) \\
= & \operatorname{var}\left(\int_{0}^{p_{s}} \nabla \cdot\left(q_{1 \pi} \vec{V}_{2 \pi}\right) d p\right)
\end{aligned}
$$

Thermodynamic (Eq. 5) and dynamic (Eq. 6) contributions to the changes of MTM under RCP4.5 scenarios are compared by constructing PDFs using $\mathbf{Y}_{\mathbf{t h m}}$ and $\mathbf{Y}_{\mathbf{d y n}}$. The emphasis will be on the tail behavior of the thermodynamic and dynamic PDFs, since the variance of statistical samples is mainly reflected in the width of a distribution curve. 


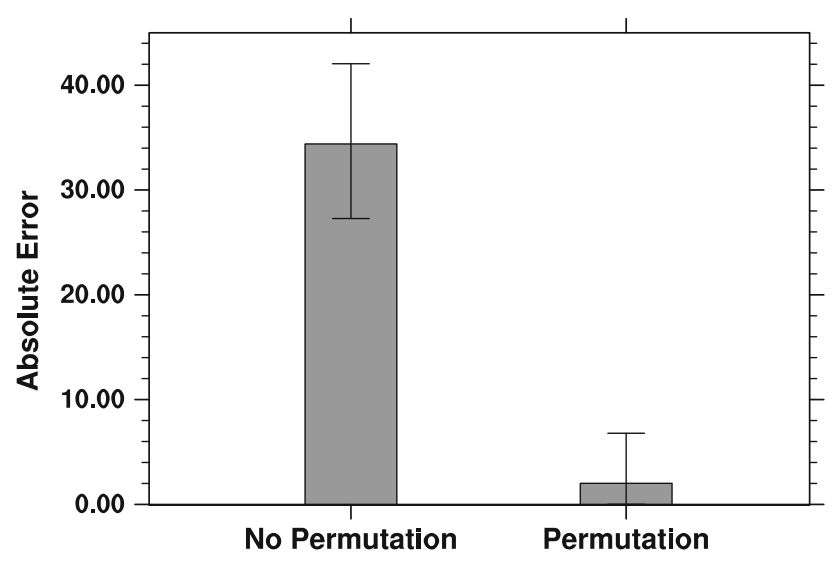

Fig. 7 Absolute errors of variance change estimation without considering the $\Delta a \cdot \Delta b$ term in Eq. (10). The bars denote the maximum likelihood estimator of absolute errors according to 1,000-time Monte-Carlo simulations; the lower and upper limits of the error bars denote the $99 \%$ uncertainty range

Figure 5 shows the PDFs constructed using $\mathbf{Y}_{\text {thm }}$ and $\mathbf{Y}_{\mathbf{d y n}}$. Compared to the MTM PDF in Historical experiment, both thermodynamic and dynamic PDFs show substantial extension of the distribution tails (Fig. 5); this result suggests that both thermodynamics and dynamics contribute to the increased MTM variance over the SE US under the RCP4.5 scenarios. Thus, the changes in SE US summer precipitation variance are not purely thermodynamic responses of regional hydrological cycle to GHGs forcing.

Specifically, the thermodynamics mainly contribute to the extension of the wet tails. Meanwhile, the mode of the thermodynamic PDF slightly shifts (although insignificant) towards increased convergence of moisture over the SE US (Fig. 5a). The shift in the PDF is consistent with the thermodynamically driven changes in hydrological cycle, with the wet regions generally getting wetter in a warming climate (e.g., Allen and Ingram 2002; Held and Soden 2006; Chou et al. 2009). Over the SE US, the thermodynamic contributions can explain the more frequent and intensified wet summers as projected by CMIP5 models ( $\mathrm{Li}$ et al. 2013a), but fail to explain the extension of the divergence tails in MTM PDFs and the enhancement of dry summers (Figs. 4f, 5a).

In contrast, atmospheric dynamics explains the enhancement of both dry and wet summers in a warming climate, via its modulation of regional hydrological cycle (Fig. 5b). Under the RCP4.5 scenarios, the tails of the dynamic PDF extend towards stronger convergence and divergence of moisture in the Southeast, which closely resembles the PDF of the RCP4.5 MTM (Fig. 5b). Compared to the thermodynamic PDFs, the dynamic contributions are particularly important in explaining future changes in summertime drought over this region (Fig. 5).
Synthetically, the changes in atmospheric dynamics exert a broader impact on the variance change of SE US regional hydrological cycle during summer seasons, which tend to result in both extremely dry and wet summers in the future.

The dynamic contributions, as quantified using the variance partition algorithm, are consistent with the pattern shift of the NASH western ridge in a warming climate. The NASH western ridge has been suggested to be one of the most important circulation systems that regulates SE US summer precipitation in current (e.g., Davis et al. 1997; Katz et al. 2003; Li et al. 2011, 2012b) and future climate (e.g., Li et al. 2011, 2013a; Wuebbles et al. 2013). In a warming climate, the NASH western ridge would extend further westward ( $\mathrm{Li}$ et al. 2011, 2013a), and the occurrence of Northwest (NW)- and Southwest (SW)-type ridges (Li et al. 2013a) would increase accordingly. Since the NW (SW)-type ridges can cause anomalous divergence (convergence) in the lower troposphere ( $\mathrm{Li}$ et al. 2012a, b, 2013b) and thus dry (wet) summers over the SE US, their increased occurrences could enhance SE US summer precipitation variance by dynamically regulating the regional hydrological cycle.

\section{Concluding remarks}

Regional precipitation variance largely determines the frequency and severity of extreme events, such as drought and floods (e.g., Katz and Brown 1992). Studies suggest that future climate change is expected to enhance precipitation variance by accelerating the hydrological cycle. Yet, diagnosing the causes of precipitation variance change remains challenging, and leads to large uncertainties in projecting extremes in future precipitation, especially at regional scales.

Our study fills this gap by formulating a variance partition algorithm and applying it to SE US summer precipitation. This algorithm utilizes the statistical characteristics of sample variance to explicitly quantify the contributions of atmospheric thermodynamics (specific humidity) and dynamics (wind) to precipitation variance change. Using SE US summertime hydroclimate as an example, the algorithm is able to (1) identify the factors and processes that constrain the skill of GCMs in simulating the variance of precipitation; and (2) explore the mechanisms responsible for future precipitation changes by quantifying the thermodynamic and dynamic contributions to precipitation variance.

Evaluation of CMIP5 model simulated SE US summertime hydroclimate suggests that GCMs underestimate the MTM variance, leading to an underestimation of SE US summer precipitation variance. The variance partition algorithm further demonstrates that the underestimated 
precipitation and MTM variances are directly associated with the biases in atmospheric dynamics (large-scale circulation). Thus, improvements in simulating the large-scale circulation are needed to achieve a better representation of regional hydroclimate over the SE US.

Among the 18 CMIP5 models analyzed in this study, six models reasonably simulate the summertime hydroclimate in the twentieth century over the SE US. They are characterized as the 1st class models and are implemented for mechanistic study of future changes in SE US summertime hydroclimate. These models collectively project an increase in SE US summer precipitation variance in a warming climate, caused by a higher variance of MTM.

According to the variance partition algorithm, the increased MTM and precipitation variances result from a combined thermodynamic and dynamic effect, with the latter being more important. Specifically, the thermodynamic contributions are manifested in the enhancement of moisture convergence and the intensification of wet summers. However, thermodynamics alone cannot explain the projected enhancement in summer drought, suggesting that the variance changes in the SE US summertime hydrological cycle are not a purely thermodynamic response to GHG forcing. In contrast, changes in atmospheric dynamics, i.e., the future changes in large-scale circulation, result in the intensification of both wet and dry summers, indicating that the dynamics explains a more complete spectrum of future changes in the SE US summertime hydrological cycle. Thus, to better understand the changes in the SE US summertime hydrological cycle, especially the intensification of summer drought, the dynamic processes should be better simulated in "state-of-the-art" models.

The projected changes in the dynamic components may result from the pattern shift of the NASH western ridge in a warming climate. As warming continues, the NASH western ridge is expected to extend further into the US continent; the Northwest and Southwest-type ridge patterns would increase accordingly. As the Northwest (Southwest)type ridge tends to enhance mass divergence (convergence) at the lower troposphere and decrease (increase) summer precipitation over the SE US (Li et al. 2013b), their increased occurrence in the future could drive SE US summer precipitation towards more extremes by dynamically regulating the regional hydrological cycle.

Acknowledgments The authors thank the international modeling groups for providing their data for analysis, the Program for Climate Model Diagnosis and Intercomparison (PCMDI) for collecting and archiving the model data, the JSC/CLIVAR Working Group on Coupled Modeling (WGCM) and their Coupled Model Intercomparison Project (CMIP) and Climate Simulation Panel for organizing the model data analysis activity. We thank Drs. Richard Seager and Kenneth Kunkel for insightful discussion; two anonymous reviewers for helpful comments; and Ms. Diane Bryson for editorial help. This work is supported by the NSF Grant AGS-1147608 and NIH1R21AG044294-01A1.

\section{Appendix 1: Summertime hydroclimate over the SE US}

On temporal scales longer than 10 days, the atmospheric branch of the regional hydrological cycle is characterized as the moisture balance between precipitation, evapotranspiration, and net moisture transport across the domain lateral boundary (Brubaker et al. 1993). Thus, SE US summertime hydrological cycle can be quantified as:

$\rho_{w} g([\bar{P}]-[\bar{E}])=-\left[\nabla \cdot \overline{\int_{0}^{p_{s}} q \vec{V} d p}\right]$

where $P$ is precipitation; $E$ is evapotranspiration; and $\nabla$. $\int_{0}^{p_{s}} q \vec{V} d p$ quantifies the net moisture transport across the domain lateral boundary (hereafter, "moisture transport (MT)" for abbreviation). The bars and [] denote the average over the JJA season and the domain average over the terrestrial SE US, respectively.

The MT in Eq. 7 can be further partitioned into: MT caused by JJA mean circulation (MTM), subseasonal-scale eddies (MTE), and surface properties (MTS), respectively (e.g. Trenberth and Guillemot 1995; Seager et al. 2010; Seager and Henderson 2013):

$$
\begin{aligned}
& \nabla \cdot \underbrace{\overline{\int_{0}^{p_{s}} q \vec{V} d p}}_{M T}=\underbrace{\int_{0}^{p_{s}} \nabla \cdot(\bar{q} \overrightarrow{\bar{V}}) d p}_{M T M}+\underbrace{\int_{0}^{p_{s}} \nabla \cdot \overline{\left(\overline{q^{\prime}} \overrightarrow{V \prime}\right)} d p}_{M T E} \\
& +\underbrace{\overline{q_{s} \vec{V}_{s} \cdot \nabla p_{s}}}_{M T S}
\end{aligned}
$$

In Eq. 8, the JJA mean (denoted by bar) is calculated using the average of 6-h (3-h for NARR) data in each summer. The primes are the $6-\mathrm{h}$ (3-h for NARR) deviation from the JJA mean.

Equations 7 and 8 indicate that the variance of SE US summer precipitation can be introduced by evapotranspiration, MT processes associated with JJA mean flow (MTM in Eq. 8), subseasonal-scale eddies (MTE in Eq. 8), and surface properties (MTS in Eq. 8: surface moisture flux across the surface pressure gradient), or the interaction of these processes. Thus, a comprehensive analysis of the regional hydrological cycle according to Eqs. 7 and 8 enables the diagnosis and quantification of the processes that contributes to SE US summer precipitation variance.

Figure 6 shows the pair-wise relationship between terms in Eqs. 7 and 8 using observed precipitation and the reanalysis ensemble. Generally, both evapotranspiration 
and MT linearly correlate with precipitation (Fig. 6). However, the contribution of MT to precipitation variance is far larger than that of evapotranspiration. The linear regression between MT and precipitation has an $R^{2}$ value of 0.64 , indicating that MT can explain the majority of SE US summer precipitation variance. In contrast, the variance explained by evapotranspiration is less than $10 \%$, suggesting its role is secondary compared to MT processes.

Further, the MTM predominates the variance of MT and precipitation. The reanalysis ensemble suggests that the MTM explains more than $90 \%$ of the MT variance, and shows a near one-to-one relationship with precipitation. Its contributions to summer precipitation outweigh that of the MTE and MTS (Fig. 6). The MTE (associated with subseasonal-scale systems, such as hurricane land fallings and frontal systems) usually contributes to extreme weather events over the SE US (e.g., Kunkel et al. 2010; Barlow 2011; Prat and Nelson 2013). However, on seasonal scales, their contributions to SE US summer precipitation variance are relatively small and nonlinear (Fig. 6).

Overall, the analysis of the atmospheric hydrological cycle suggests that the variance of SE US summer precipitation is primarily controlled by the variance of MT, while evapotranspiration is secondary. Furthermore, most MT variance $(90 \%)$ can be explained by the MTM, indicating the importance of the seasonal mean circulation in regulating the variance of SE US summer precipitation.

\section{Appendix 2: Variance change partition without permutation}

Without permutation, the MTM variance in state 1 to state 2 are expressed as $\operatorname{var}\left(\mathbf{Y}_{\mathbf{1}}\right)=\operatorname{var}\left(\int_{0}^{p_{s}} \nabla \cdot\left(q_{1} \vec{V}_{1}\right) d p\right)$, and $\operatorname{var}\left(\mathbf{Y}_{2}\right)=\operatorname{var}\left(\int_{0}^{p_{s}} \nabla \cdot\left(q_{2} \vec{V}_{2}\right) d p\right)$, respectively. Accordingly, the changes of thermodynamic components (specific humidity) between state 1 and state 2 are $\Delta q=q_{2}-q_{1}$, and the changes of dynamic components (wind) are $\Delta \vec{V}=\vec{V}_{2}-\vec{V}_{1}$. Thus, the changes of MTM variance in state 2 can be expressed as:

$$
\begin{aligned}
\operatorname{var}\left(\mathbf{Y}_{2}\right)= & \operatorname{var}\left(\int_{0}^{p_{s}} \nabla \cdot\left(q_{1} \vec{V}_{1}\right) d p+\int_{0}^{p_{s}} \nabla \cdot\left(\Delta q \vec{V}_{1}\right) d p\right. \\
& \left.+\int_{0}^{p_{s}} \nabla \cdot\left(q_{1} \Delta \vec{V}\right) d p+\int_{0}^{p_{s}} \nabla \cdot(\Delta q \Delta \vec{V}) d p\right)
\end{aligned}
$$

Unlike the term $\int_{0}^{p_{s}} \nabla \cdot\left(\Delta q_{\pi} \Delta \vec{V}_{\pi}\right) d p$ in Eq. (1), the term $\int_{0}^{p_{s}} \nabla \cdot(\Delta q \Delta \vec{V}) d p$ in Eq. (9) cannot be neglected because $\Delta q$ and $\Delta \vec{V}$ is not necessarily a small deviation from the original state i.e., state 1 . To verify this, we use hypothetical Monte-Carlo samples as examples. Assume $\mathbf{Y}_{\mathbf{1}}=a_{1} \cdot b_{1}$, and $\mathbf{Y}_{\mathbf{2}}=a_{2} \cdot b_{2}$. We draw 1,000 samples of both $\mathrm{a}_{1}$ and $\mathrm{b}_{1}$ from $N(10,0.8)$. In state 2 , we keep $\mathrm{a}_{2}$ the same as $\mathrm{a}_{1}$, but draw $\mathrm{b}_{2}$ from $N(12,1.0)$. Thus the variance difference between $\mathbf{Y}_{\mathbf{2}}$ and $\mathbf{Y}_{\mathbf{1}}$ is:

$$
\begin{aligned}
\delta \operatorname{var}= & \operatorname{var}\left(\mathbf{Y}_{\mathbf{2}}\right)-\operatorname{var}\left(\mathbf{Y}_{\mathbf{1}}\right) \\
= & \operatorname{var}\left(a_{1} \cdot b_{1}+\Delta a \cdot b_{1}+a_{1} \cdot \Delta b+\Delta a \cdot \Delta b\right) \\
& -\operatorname{var}\left(a_{1} \cdot b_{1}\right)
\end{aligned}
$$

According to the 1000-time Monte-Carlo simulations of $\mathrm{Y}_{1}$ and $\mathrm{Y}_{2}$, the maximum likelihood estimator (MLE) of $\delta$ var is 83.81 .

Neglecting $\Delta a \cdot \Delta b$ on the right-hand side of Eq. (10), then the estimated variance change would be $\delta \operatorname{var}_{\text {est }}=\operatorname{var}\left(a_{1} \cdot b_{1}+\Delta a \cdot b_{1}+a_{1} \cdot \Delta b\right)-\operatorname{var}\left(a_{1} \cdot b_{1}\right)$. Without permutation, the absolute error of $\delta \mathrm{var}_{\text {est }}$ in comparison with $\delta$ var is 34.40 on average, which is equivalent to $41 \%$ of the total variance change (Fig. 7). It indicates that $\Delta a \cdot \Delta b$ makes substantial contributions to variance change, and thus cannot be neglected in Eq. (10) without permutation.

Keeping $\Delta a \cdot \Delta b$ in Eq. (10), $\delta$ var is expressed as:

$$
\begin{aligned}
\delta \operatorname{var}= & \operatorname{var}\left(a_{1} \cdot b_{1}+\Delta a \cdot b_{1}+a_{1} \cdot \Delta b+\Delta a \cdot \Delta b\right)-\operatorname{var}\left(a_{1} \cdot b_{1}\right) \\
= & \operatorname{var}\left(\Delta a \cdot b_{1}\right)+\operatorname{var}\left(a_{1} \cdot \Delta b\right)+\operatorname{var}(\Delta a \cdot \Delta b) \\
& +2 \operatorname{cov}\left(a_{1} \cdot b_{1}, \Delta a \cdot b_{1}\right)+2 \operatorname{cov}\left(a_{1} \cdot b_{1}, a_{1} \cdot \Delta b\right) \\
& +2 \operatorname{cov}\left(a_{1} \cdot b_{1}, \Delta a \cdot \Delta b\right) \\
& +2 \operatorname{cov}\left(\Delta a \cdot b_{1}, a_{1} \cdot \Delta b\right)+2 \operatorname{cov}\left(\Delta a \cdot b_{1}, \Delta a \cdot \Delta b\right) \\
& +2 \operatorname{cov}\left(\Delta a \cdot b_{1}, a_{1} \cdot \Delta b\right)
\end{aligned}
$$

In Eq. (11), 4 additional terms are added compared to Eq. (3); that are $\operatorname{var}(\Delta a \cdot \Delta b), 2 \operatorname{cov}\left(a_{1} \cdot b_{1}, \Delta a \cdot \Delta b\right)$, $2 \operatorname{cov}\left(\Delta a \cdot b_{1}, \Delta a \cdot \Delta b\right)$, and $2 \operatorname{cov}\left(a_{1} \cdot \Delta b, \Delta a \cdot \Delta b\right)$. These four terms involve the interaction between $\Delta a$ and $\Delta b$, which make their contributions to $\delta$ var inseparable.

In contrast, after permutation, $\mathbf{Y}_{1 \pi}=a_{1 \pi} \cdot b_{1 \pi}$, and $\mathbf{Y}_{2 \pi}=a_{2 \pi} \cdot b_{2 \pi} . \Delta a_{\pi}=a_{2 \pi}-a_{1 \pi}$ and $\Delta b_{\pi}=b_{2 \pi}-b_{1 \pi}$ are small deviation from $a_{1 \pi}$ and $b_{1 \pi}$, respectively. Thus, they can be neglected in Eq. (10) without introducing significant errors. Neglecting $\Delta a_{\pi} \cdot \Delta b_{\pi}$, the variance change can be approximated as $\delta \operatorname{var}_{e s t} \pi=\operatorname{var}\left(a_{1 \pi} \cdot b_{1 \pi}+\Delta a_{\pi}\right.$. $\left.b_{1 \pi}+a_{1 \pi} \cdot \Delta b_{\pi}\right)-\operatorname{var}\left(a_{1 \pi} \cdot b_{1 \pi}\right)$.

According to the Monte-Carlo simulations of hypothetical time series, the errors introduced by neglecting the $\Delta a_{\pi} \cdot \Delta b_{\pi}$ term in the permutation case is only 2.02 ( $\sim 2.4 \%$ relative error), which is substantially smaller than that without permutation (Fig. 7). It indicates that after permutation, the variance partition can be simplified by neglecting the $\Delta a_{\pi} \cdot \Delta b_{\pi}$ term. Applying the permutation, as described in Sect. 3, to MTM time series, the contributions of thermodynamic and dynamics to MTM variance change can be quantified as in Eq. (4). 


\section{References}

Allen MR, Ingram WJ (2002) Constraints on future changes in the hydrological cycle. Nature 419:224-232

Barlow M (2011) Influence of hurricane-related activity on North American extreme precipitation. Geophys Res Lett 38:L04705

Bolstad BM, Irizarry RA, Astrand M, Speed TP (2003) A comparison of normalization methods for high density oligonucleotide array data based on variance and bias. Bioinformatics 19:185-193

Brubaker KL, Entekhabi D, Eagleson PS (1993) Estimation of continental precipitation recycling. J Clim 6:1077-1089

Cai W, Cowan T, Thatcher M (2012) Rainfall reductions over Southern Hemisphere semi-arid regions: the role of subtropical dry zone expansion. Sci Rep 2:702

Chen M, Xie P, Janowiak JE, Arkin PA (2002) Global land precipitation: a 50-year monthly analysis based on gauge observations. J. Hydrometeorol 3:249-266

Chou C, Neelin JD, Chen C-A, Tu J-Y (2009) Evaluating the "RichGet-Richer" mechanism in tropical precipitation change under global warming. J Clim 22:1982-2005

Clark CO, Cole JE, Webster PJ (2000) Indian Ocean SST and Indian summer rainfall: predictive relationships and their decadal variability. J Clim 13:2503-2519

Dai A (2006) Recent climatology, variability, and trends in global surface humidity. J Clim 19:3589-3606

Dairaku K, Emori S (2006) Dynamic and thermodynamic influences on intensified daily rainfall during the Asian summer monsoon under doubled atmospheric $\mathrm{CO}_{2}$ conditions. Geophys Res Lett 33:L01704

Davis RE, Hayden BP, Gay DA, Phillips WL, Jones GV (1997) The North Atlantic subtropical anticyclone. J Clim 10:728-744

DeGroot MH, Schervish MJ (2011) Probability and statistics. Addison Wesley Publishing Company Incorporated, Reading, MA

Feng S, Fu Q (2013) Expansion of global drylands under a warming climate. Atmos Chem Phys 13:10081-10094

Feng L, Zhou T (2012) Water vapor transport for summer precipitation over the Tibetan Plateau: multi-dataset analysis. J Geophys Res Atmos 117:D20114

Giorgi F, Francisco R (2000) Uncertainties in regional climate change predictions: a regional analysis of ensemble simulations with the HadCM2 GCM. Clim Dyn 16:169-182

Gotvald AJ, McCallum BE (2010) Epic flooding in Georgia, 2009: US geological survey fact sheet 2010-3107

Held IM, Soden BJ (2006) Robust responses of the hydrological cycle to global warming. J Clim 19:5686-5699

Higgins RW, Shi W, Yarosh E, Joyce R (2000) Improved United States precipitation quality control system and analysis. NCEP/ Climate Prediction Center ATLAS No. 7, Camp Springs, MD 20746, USA

Hoff PD (2009) A first course in Bayesian statistical methods, Springer texts in statistics. Springer, New York

Huntington TG (2006) Evidence for intensification of the global water cycle: review and synthesis. J Hydrol 319(1-4):83-95

Kalnay E et al (1996) The NCEP-NCAR 40-year reanalysis project. Bull Am Meteorol Soc 77:437-471

Karl TR, Knight RW (1998) Secular trends of precipitation amount, frequency, and intensity in the United States. Bull Am Meteorol Soc 79:231-241

Katz RW, Brown BG (1992) Extreme events in a changing climate: variability is more important than averages. Clim Change 21:289-302

Katz RW, Parlange MB, Tebaldi C (2003) Stochastic modeling of the effects of large-scale circulation on daily weather in the southeastern US. Clim Change 60:189-216
Kawase H, Abe M, Yamada Y, Takemura T, Yokohata T, Nozawa T (2010) Physical mechanism of long-term drying trend over tropical North Africa. Geophys Res Lett 37:L09706

Kharin VV, Zwiers FW (2000) Changes in the extremes in an ensemble of transient climate simulations with a coupled atmosphere-ocean GCM. J Clim 13:3760-3788

Kitoh A, Endo H, Kumar KK, Cavalcanti IFA, Goswami P, Zhou T (2013) Monsoons in a changing world: a regional perspective in a global context. J Geophys Res Atmos 118:3053-3065

Konrad CE (1997) Synoptic-scale features associated with warm season heavy rainfall over the interior southeastern United States. Weather Forecast 12:557-571

Kunkel KE, Easterling D, Kristovich DAR, Gleason B, Stoecker L, Smith R (2010) Recent increases in U.S. heavy precipitation associated with tropical cyclones. Geophys Res Lett 37:L24706

Kushnir Y, Seager R, Ting M, Naik N, Nakamura J (2010) Mechanisms of tropical Atlantic SST influence on North American precipitation variability. J Clim 23:5610-5628

Lau WK-M, Wu H-T, Kim K-M (2013) A canonical response of precipitation characteristics to global warming from CMIP5 models. Geophys Res Lett 40:3163-3169

Li W, Li L, Fu R, Deng Y, Wang H (2011) Changes to the North Atlantic subtropical high and its role in the intensification of summer rainfall variability in the Southeastern United States. J Clim 24:1499-1506

Li L, Li W, Kushnir Y (2012a) Variation of North Atlantic subtropical high western ridge and its implication to the Southeastern US summer precipitation. Clim Dyn 39:1401-1412

Li W, Li L, Ting M, Liu Y (2012b) Intensification of Northern Hemisphere subtropical highs in a warming climate. Nat Geosci 5:830-834

Li L, Li W, Deng Y (2013a) Summer rainfall variability over the Southeastern United States in the 21 st century as assessed by the CMIP5 models. J Geophys Res Atmos 118:340-354

Li L, Li W, Barros AP (2013b) Atmospheric moisture budget and its regulation of the summer precipitation variability over the Southeastern United States. Clim Dyn 41:613-631

Lin R, Zhou T, Qian Y (2014) Evaluation of global monsoon precipitation changes based on five reanalysis datasets. J Clim 27:1271-1289

Mahajan S, North GR, Saravanan R, Genton MG (2012) Statistical significance of trends in monthly heavy precipitation over the US. Clim Dyn 38:1375-1387

Manuel J (2008) Drought in the southeast: lessons for water management. Environ Health Perspect 116:A168-A171

Martinez CJ, Baigorria GA, Jones JW (2009) Use of climate indices to predict corn yields in southeast USA. Int J Climatol 29:1680-1691

Mesinger F et al (2006) North American regional reanalysis. Bull Am Meteorol Soc 87:343-360

Moss RH et al (2010) The next generation of scenarios for climate change research and assessment. Nature 463:747-756

O'Gorman PA, Schneiderb T (2009) The physical basis for increases in precipitation extremes in simulations of 21st-century climate change. Proc Natl Acad Sci 106:14773-14777

Onogi K et al (2007) The JRA-25 reanalysis. J Meteorol Soc Japan 85:369-432

Prat OP, Nelson BR (2013) Precipitation contribution of tropical cyclones in the Southeastern United States from 1998 to 2009 using TRMM satellite data. J Clim 26:1047-1062

Rhee J, Im J, Carbone GJ, Jensen JR (2008) Delineation of climate regions using in situ and remotely-sensed data for the Carolinas. Remote Sens Environ 112:3099-3111

Rudolf BC, Beck C, Grieser J, Schneider U (2005) Global precipitation analysis products. Global Precipitation Climatology Centre (GPCC), DWD, Internet publication, pp 1-8 
Santer BD et al (2007) Identification of human-induced changes in atmospheric moisture content. Proc Natl Acad Sci 104: $15248-15253$

Scheff J, Frierson DMW (2012) Robust future precipitation declines in CMIP5 largely reflect the poleward expansion of model subtropical dry zones. Geophys Res Lett 39:L18704

Seager R, Henderson N (2013) Diagnostic computation of moisture budgets in the ERA-Interim reanalysis with reference to analysis of CMIP-archived atmospheric model data. J Clim 26:7876-7901

Seager R et al (2007) Model projections of an imminent transition to a more arid climate in Southwestern North America. Science 316:1181-1184

Seager R, Naik N, Vecchi GA (2010) Thermodynamic and dynamic mechanisms for large-scale changes in the hydrological cycle in response to global warming. J Clim 23:4651-4668

Seager R, Naik N, Vogel L (2012) Does global warming cause intensified interannual hydroclimate variability? J Clim 25:3355-3372

Sherwood SC, Roca R, Weckwerth TM, Andronova NG (2010) Tropospheric water vapor, convection and climate. Rev Geophys 48:2001

Skific N, Francis JA, Cassano JJ (2009) Attribution of seasonal and regional changes in Arctic moisture convergence. J Clim 22:5115-5134

Taylor KE, Stouffer RJ, Meehl GA (2009) A summary of the CMIP5 experiment design, PCMDI Rep., p 33. http://cmip.PCMDI.1lnl. gov/cmip5/doc/Taylor_CMIP5_design.pdf
Taylor KE, Stouffer RJ, Meehl GA (2012) An overview of CMIP5 and the experiment design. Bull Am Meteorol Soc 93:485-498

Trenberth KE (2011) Changes in precipitation with climate change. Clim Res 47:123-138

Trenberth KE, Guillemot CJ (1995) Evaluation of the global atmospheric moisture budget as seen from analyses. J Clim 8:2255-2272

Trenberth KE, Dai A, Rasmussen RM, Parsons DB (2003) The changing character of precipitation. Bull Am Meteorol Soc 84:1205-1217

Uppala SM et al (2005) The ERA-40 re-analysis. Q J R Meteorol Soc 131:2961-3012

Vecchi GA, Soden BJ, Wittenberg AT, Held IM, Leetmaa A, Harrison MJ (2006) Weakening of tropical Pacific atmospheric circulation due to anthropogenic forcing. Nature 441:73-76

Wang H, Fu R, Kumar A, Li W (2010) Intensification of summer rainfall variability in the southeastern United States during recent decades. J Hydrometeorol 11:1007-1018

Wuebbles D et al (2013) CMIP5 climate model analyses: climate extremes in the United States. Bull Am Meteorol Soc. doi:10. 1175/BAMS-D-12-00172.1

Yang F, Kumar A, Schlesinger ME, Wang W (2003) Intensity of hydrological cycles in warming climate. J Clim 16:2419-2423

Zhou T, Yu R (2005) Atmospheric water vapor transport associated with typical anomalous summer rainfall patterns in China. J Geophys Res Atmos 110:D08104 